\title{
Renin-Angiotensin-Aldosterone System Inhibitors and Risk of Death in Patients Hospitalised with COVID-19: A Retrospective Italian Cohort Study of 43,000 Patients
}

\author{
Gianluca Trifiròn $^{1,2}$ (1) Marco Massari $^{3} \cdot{\text { Roberto Da } \text { Cas }^{3} \cdot \text { Francesca Menniti Ippolito }^{3} \cdot \text { Janet Sultana }}^{1,2}$. \\ Salvatore Crisafulli ${ }^{1,2}$. Paolo Giorgi Rossi ${ }^{4}$. Massimiliano Marino ${ }^{4}$. Manuel Zorzi ${ }^{5}$ Emanuela Bovo ${ }^{5}$. Olivia Leoni ${ }^{6}$. \\ Monica Ludergnani ${ }^{6}$. Stefania Spila Alegiani ${ }^{3} \cdot$ ITA-COVID-19: RAAS inhibitor group
}

Published online: 27 August 2020

(c) Springer Nature Switzerland AG 2020

\begin{abstract}
Introduction The epidemic due to severe acute respiratory syndrome coronavirus 2 (SARS-CoV-2) infection has been spreading globally, raising increasing concerns. There are several controversial hypotheses on the potentially harmful or beneficial effects of antihypertensive drugs acting on the renin-angiotensin-aldosterone system (RAAS) in coronavirus disease 2019 (COVID-19). Furthermore, there is accumulating evidence, based on several observational studies, that angiotensinconverting enzyme inhibitors (ACEIs) and angiotensin receptor blockers (ARBs) do not increase the risk of contracting SARS-CoV-2 infection. On the other hand, conflicting findings regarding the role of ACEIs/ARBs as prognosis modifiers in COVID-19 hospitalised patients have been reported.

Objective The aim of this large-scale, retrospective cohort study was to investigate whether prior exposure to ACEIs and/or ARBs was associated with all-cause mortality among over 40,000 hospitalised COVID-19 patients compared with calcium channel blockers (CCBs), a potential therapeutic alternative.

Methods This study was conducted using COVID-19 registries linked to claims databases from Lombardy, Veneto and Reggio Emilia (overall, 25\% of Italian population). Overall, 42,926 patients hospitalised between 21 February and 21 April 2020 with a diagnosis of COVID-19 confirmed by real-time polymerase chain reaction tests were included in this study. All-cause mortality occurring in or out of hospital, as reported in the COVID-19 registry, was estimated. Using Cox models, adjusted hazard ratios (HRs) of all-cause mortality (along with 95\% confidence intervals [CIs]) were estimated separately for ACEIs/ARBs and other antihypertensives versus CCBs and non-use.

Results Overall, 11,205 in- and out-of-hospital deaths occurred over a median of 24 days of follow-up after hospital admission due to COVID-19. Compared with CCBs, adjusted analyses showed no difference in the risk of death among ACEI (HR $0.97,95 \%$ CI 0.89-1.06) or ARB (HR 0.98, 95\% CI 0.89-1.06) users. When non-use of antihypertensives was considered as a comparator, a modest statistically significant increase in mortality risk was observed for any antihypertensive use. However, when restricting to drugs with antihypertensive indications only, these marginal increases disappeared. Sensitivity and subgroup analyses confirmed our main findings.

Conclusions ACEI/ARB use is not associated with either an increased or decreased risk of all-cause mortality, compared with CCB use, in the largest cohort of hospitalised COVID-19 patients exposed to these drugs studied to date. The use of
\end{abstract}

Gianluca Trifirò and Marco Massari contributed equally to this article.

The members of ITA-COVID-19: RAAS inhibitor group are listed in acknowledgements.

Electronic supplementary material The online version of this article (https://doi.org/10.1007/s40264-020-00994-5) contains supplementary material, which is available to authorized users.

Extended author information available on the last page of the article 
these drugs therefore does not affect the prognosis of COVID-19. This finding strengthens recommendations of international regulatory agencies about not withdrawing/switching ACEI/ARB treatments to modify COVID-19 prognosis.

\section{Key Points}

There is conflicting preclinical evidence on whether angiotensin-converting enzyme inhibitors (ACEIs) or angiotensin receptor blockers (ARBs) increase susceptibility to severe acute respiratory syndrome coronavirus 2 (SARS-CoV-2) infection, although both hypotheses are biologically plausible.

There is also conflicting evidence on whether the use of ACEI/ARBs affects the prognosis of hospitalised patients with coronavirus disease 2019 (COVID-19).

This study found no difference in the risk of mortality associated with prior exposure to ACEIs or ARBs compared with calcium channel blockers.

Prior use of ACEIs or ARBs does not modify prognosis in COVID-19 hospitalised patients.

\section{Introduction}

Severe acute respiratory syndrome coronavirus 2 (SARS$\mathrm{CoV}-2$ ) is responsible for the global coronavirus disease 2019 (COVID-19) pandemic that has led to more than 11 million infected patients and almost 540,000 deaths worldwide as at 7 July 2020 [1]. Italy has been facing one of the largest outbreaks, with around 34,869 deaths currently [2].

There are several controversial hypotheses on the potentially harmful or beneficial effects of antihypertensive drugs acting on the renin-angiotensin-aldosterone system (RAAS) in COVID-19 [3-5]. These hypotheses are based on the fact that angiotensin-converting enzyme 2 (ACE2) is the receptor binding site for SARS-CoV-2 in the target cell [6]. In vitro and in vivo studies have demonstrated that ACE inhibitors (ACEIs), as well as angiotensin-II receptor blockers (ARBs), can significantly increase ACE2 expression, thereby facilitating SARS-CoV-2 entry into cells [7, 8]. Mechanistically, it is possible that ACE2 tissue level changes in response to ACEIs/ARBs in humans, but large clinical studies have not yet confirmed this. However, it has also been found that viral binding to ACE2 decreases its surface expression and prevents angiotensin-II cleavage by ACE to generate angiotensin 1-7, which counterbalances the effect of angiotensin-II signalling through angiotensin receptor type-1 (AT1R). Hence, binding of angiotensin-II to AT1R leads to increased pulmonary vascular permeability, resulting in lung injury $[9,10]$. By blocking AT1R-mediated angiotensin-II adverse effects and increasing ACE2-mediated production of angiotensin 1-7 production, ARBs may counteract this effect and reduce lung damage [3].

Based on several observational studies (Online Resource 1), there is accumulating evidence that ACEIs and ARBs do not increase the risk of contracting SARS-CoV-2 infection [11, 12]. On the other hand, conflicting findings regarding the role of ACEIs/ARBs as prognosis modifiers in COVID-19 hospitalised patients have been reported. Published studies on this research question suffer from small sample size and potential residual confounding as they recruited small cohorts of hospitalised patients. Small sample sizes also do not permit sensitivity analyses to be conducted, including dose-effect analyses, which can corroborate the main findings [13-17]. Furthermore, the identification of all covariates related to prior use of chronic medications, as well as comorbidities and a history of prior hospitalisations, may not be accurately evaluated in this setting during a healthcare emergency if data are collected prospectively. The mixed quality of studies investigating whether ACEI/ARB use modifies the prognosis of COVID-19 is seen by the fact that two meta-analyses of observational studies reported contrasting results. One meta-analysis suggested a protective effect of RAAS inhibitors regarding all-cause mortality and critical illness [18], while the other found no significant association with all-cause mortality [19]. In addition to the contradictory results, these meta-analyses are problematic because they included an observational study that has since been retracted [20]. This highlights the need for a large-scale study using appropriate methods to investigate whether prior ACEI/ARB use among persons with COVID-19 improves or worsens the prognosis of persons infected with SARS-CoV-2. Ascertaining the impact of these drugs is essential because it has been suggested that withdrawing RAAS inhibitors and switching to other antihypertensives, such as calcium channel blockers (CCBs), may be beneficial [21], but there is very little evidence emerging from direct comparisons between these two drug classes [11, 14, 22-24], limiting the usefulness of available studies in clinical practice.

The aim of this population-based Italian retrospective cohort study was to investigate whether ACEI/ARB use prior to hospitalisation increases or decreases the risk of death in a population of over 40,000 patients after they were hospitalised with a diagnosis of COVID-19, identified from a catchment population of 15.2 million persons in the largest study to date. 


\section{Methods}

A large-scale, retrospective cohort study was conducted in the Lombardy and Veneto regions and the Reggio Emilia Local Health Unit, covering an underlying population of 15.2 million persons ( $25 \%$ of the Italian population) overall. The protocol was registered in the ENCePP EU-PAS register (EUPAS34541).

\subsection{Data Source}

Italy has a National Health System (NHS) that provides universal care. Healthcare services provided are collected using claims databases that have been widely used for clinical and pharmacoepidemiological research [25]. The data used in this study were recorded through routine data collection, using national data collection systems that were in place prior to the start of the study. The following claims databases were used: hospital admissions, pharmacy claims and copayment exemptions. Pharmacy claims databases were updated as of 31 December 2019, 30 November 2019 and 29 February 2020 in Lombardy, Veneto and Reggio Emilia, respectively. All claims databases date back over 10 years. Hospital admissions in the 10 years preceding COVID-19 hospitalisations, as well as pharmacy claims in the past year, were extracted and used to adjust for confounding. Diagnoses were recorded using the International Classification of Diseases, Clinical Modification, 9th Revision (ICD-CM-9). Pharmacy claims in the last available 3 months prior to COVID-19-related hospitalisation were used to assess antihypertensive exposure. Drug data were recorded using the Anatomical Therapeutic Chemical (ATC) classification system and the National Drug Code (NDC). Copayment exemption databases were used as an additional source to identify diseases that exempt patients from healthcare copay. Using unique anonymised patient identifiers, claims databases were all linked locally to the COVID-19 surveillance database available in each catchment area. This registry contains information (e.g. hospitalisation date, intensive care unit [ICU] admission, death) on patients testing positive for SARS-CoV-2 RNA by polymerase chain reaction (PCR) on nasopharyngeal/throat swabs. This database has been used to identify the study population and outcome, and is updated up to 21 April 2020 for Lombardy, and 31 March 2020 for Veneto and Reggio Emilia.

An R-based tool for distributed analyses developed by the Italian National Institute of Health (The ShinISS) was employed by each centre to locally elaborate COVID-19 patient data using a common data model, sharing only a fully anonymised dataset for central analysis, in compliance with EU General Data Protection Regulation regulations.

\subsection{Study Population}

All patients aged $\geq 18$ years with SARS-CoV-2 infection who were hospitalised from 21 February (date of the first confirmed Italian COVID-19 patient hospitalisation) to 21 April 2020 were identified in the three centres, from the local COVID-19 registry. Admission date was considered the index date (ID). Included patients were followed-up until death (occurring in any setting) or end of the observation period, whichever came first.

\subsection{Exposure Definition}

Exposure of interest was any antihypertensive drug, grouped, using pharmacy claims, into the following mutually exclusive categories: (1) ACEI as monotherapy or fixed combination with other antihypertensive drugs, except for ARBs and CCBs; (2) ACEIs + CCBs as fixed or non-fixed combination; (3) ARBs as monotherapy or fixed combinations with other antihypertensive drugs, except for ACEIs and CCBs; (4) ARBs + CCBs as fixed or non-fixed combinations; (5) CCBs as monotherapy or fixed combination with other antihypertensives, except for ACEIs or ARBs; (6) other antihypertensive drugs, including $\alpha 2$-adrenergic receptor agonists, $\beta$-blockers and diuretics; and (7) non-use of any antihypertensive drug (Online Resource 2). A patient was considered exposed to each of the above-mentioned categories if they had at least one pharmacy claim within 3 months prior to the ID. The ACEI, ARB or CCB categories also included a non-fixed combination with drugs from other antihypertensive categories.

\subsection{Outcomes}

The study outcome was all-cause mortality occurring in or out of hospital, as reported in the COVID-19 registry.

\subsection{Potential Confounders}

In addition to sex, age and catchment area, the following potential confounders were identified: number of hospitalisations in the last 2 years and drug dispensing in the last year; Charlson index (Online Resource 3); cardiocerebrovascular diseases, as well as other acute and chronic diseases; prior use of several chronically used drugs and recent use of corticosteroids, nonsteroidal anti-inflammatory drugs (NSAIDs), and chloroquine/hydroxychloroquine. Information on diseases was extracted from copayment exemption and hospital discharge databases within the last 10 years of data availability (Online Resource 4). Prior and recent drug use was assessed using pharmacy claims within the last available year and 3 months, respectively (Online Resource 5). 


\subsection{Statistical Analysis}

Categorical and continuous variables were described using frequencies with percentages and medians with interquartile ranges (IQRs), respectively. Mortality risk following COVID-19-related hospitalisation was estimated as hazard ratios (HRs) along with 95\% confidence intervals (CIs), using a mixed-effect Cox proportional hazard model, comparing ACEIs, ARBs, and other antihypertensives versus CCBs. In an additional analysis, risk was estimated considering non-use of any antihypertensive as the reference category. We modelled catchment area as a random effect. Potential confounders were included in the multivariable model using a stepwise Akaike information criterion (AIC) method. Proportional hazard assumptions were verified on the basis of Schoenfeld residuals, and significance was set at a $p$ value $<0.05$. All statistical analyses were performed using STATA version 16 (StataCorp LLC, College Station, TX, USA) and R version 3.6 (The R Foundation for Statistical Computing, Vienna, Austria).

\subsection{Subgroup and Sensitivity Analyses}

First, we estimated mortality risk using a propensity-score (PS) matched (1:1) analysis (Online Resources 6 and 7). The standardised differences (SDs) between the exposure groups for each covariate were estimated (Online Resource 8). The nearest-neighbour matching algorithm was used (calliper width 0.05 of the standard deviation of the logit score). Second, to control for potential confounding effects of diuretics and other antihypertensives from the ACEI, ARB and CCB categories, we removed those patients receiving non-fixed combinations of other antihypertensives. Third, we assessed risk estimates only among incident users of antihypertensives (i.e. treatment started within the last 3 months prior to the ID, with no use within 9 months prior). We also explored mortality risk, stratified by any of the diseases listed among the approved treatment indications (hypertension, heart failure, acute myocardial infarction and diabetes mellitus as a proxy of diabetic nephropathy) and cumulative antihypertensive exposure in the last year (categorised by tertile of the total number of defined daily doses (DDDs), with the first tertile being the lowest cumulative exposure and the third tertile being the highest). The number of DDDs was calculated as the dose of drug dispensed multiplied by the quantity dispensed, and dividing this product by the DDD for that drug (obtained from www. whocc.no/atc_ddd_index/). Mortality risk was also estimated considering only deaths occurring during hospitalisation. As different management of COVID-19 patients and pressure on healthcare system in various regions may affect mortality risk, we stratified analyses by catchment area. Finally, we explored the risk in different calendar periods to account for the centrespecific heterogeneous length of follow-up and changes in COVID-19 management over time.

\section{Results}

Overall, 42,926 hospitalised COVID-19 patients were included in the study (Table 1), with Lombardy accounting for $90.2 \%$ of these patients (Online Resource 9). Patients' median age was 69 years (IQR 57-79), with a male-tofemale ratio of 1.67. The proportion of COVID-19 patients admitted to the ICU, including antihypertensive users and non-users, during the hospital stay ranged from $7.6 \%$ to $18.2 \%$ in the three catchment areas. In total, 11,205 (26.1\%) of all patients died over a median follow-up of 24 days (IQR 10-35) (Table 2). The total number of patients who were still hospitalised was 8638 (20.1\%), while 22,828 (53.2\%) were discharged.

Almost $50 \%$ of patients had at least one antihypertensive drug claim within 3 months prior to the ID. ARB, ACEI and CCB users accounted for $11.3 \%, 10.9 \%$ and $5.1 \%$ of total patients, respectively. The median age of antihypertensive users was 75 years, which was comparable across classes, and was much higher than non-users (61 years; IQR 51-73) (Table 1). Most antihypertensive users were male. $\mathrm{CCB}$ and other antihypertensive users more commonly had two or more hospitalisations (21-26\%) prior to the ID, and a Charlson index $\geq 3$ (13.7-17.3\%), compared with ACEI/ ARB users. A very high proportion of cardiovascular diseases and drugs among antihypertensive drug users was observed. Categories of other antihypertensives had the most severe pattern of comorbidities. Overall, burden of disease and prior use of other drugs was much higher among antihypertensive users than non-users.

In univariate analyses, advanced age was the strongest predictor of death (Table 2). Male sex, Charlson comorbidity index $\geq 1$, and most diseases, especially cardiovascular and neurodegenerative diseases (and related medications), were strongly associated with an increased risk of death. Overall, during the follow-up, the proportion of deaths among antihypertensive users ranged from 31.7 to $40.2 \%$ versus $17.1 \%$ among non-users (Table 3 ). In comparison with CCBs, unadjusted analyses showed a statistically significant protective effect for ACEIs and ARBs (also as a fixedcombination with CCB), while an increased risk for other antihypertensive drugs was reported. These statistically significant effects disappeared after adjusting for potential confounders for ACEI users (HR 0.97, 95\% CI 0.89-1.06), ARB users (HR 0.98, 95\% CI 0.89-1.06), ACEI + CCB users (HR 0.97, 95\% CI 0.90-1.05), ARB + CCB users (HR 1.02, 95\% CI 0.94-1.11), and other antihypertensives (HR 1.05, 95\% CI 0.96-1.14). In comparison with non-use, a statistically significant $2 / 2.5$-fold increased mortality risk was seen for ACEI, ARB and CCB use in unadjusted analysis. In multivariable analysis, this effect almost completely disappeared, although risk estimates were still marginally significant 
Table 1 Demographic and clinical characteristics of COVID-19 patients included in the study, by exposure group within the last 3 months of observation

\begin{tabular}{|c|c|c|c|c|c|c|c|c|}
\hline & $\begin{array}{l}\text { All COVID-19 } \\
\text { patients }\end{array}$ & $\begin{array}{l}\text { No anti-HT } \\
\text { use }\end{array}$ & ARB use & ACEI use & CCB use & $\begin{array}{l}\mathrm{ARB}+\mathrm{CCB} \\
\text { use (fixed } \\
\text { or non-fixed } \\
\text { combination) }\end{array}$ & $\begin{array}{l}\text { ACEI + CCB } \\
\text { use (fixed or } \\
\text { non-fixed com- } \\
\text { bination) }\end{array}$ & $\begin{array}{l}\text { Other anti-HT } \\
\text { use }\end{array}$ \\
\hline$N(\%)^{\mathrm{a}}$ & 42,926 & $21,974(51.2)$ & 4859 (11.3) & $4663(10.9)$ & $2178(5.1)$ & $2318(5.4)$ & $2609(6.1)$ & $4068(9.5)$ \\
\hline $\begin{array}{l}\text { Age, years } \\
{[\text { median }(\mathrm{IQR})]}\end{array}$ & $69(57-79)$ & $61(51-73)$ & $73(65-81)$ & $75(65-82)$ & $77(68-83)$ & $74(67-81)$ & $75(66-81)$ & $77(68-84)$ \\
\hline \multicolumn{9}{|l|}{ Age, years $[n(\%)]$} \\
\hline $18-49$ & $5561(13.0)$ & $5031(22.9)$ & $126(2.6)$ & $114(2.4)$ & $52(2.4)$ & $54(2.3)$ & $70(2.7)$ & $108(2.7)$ \\
\hline $50-59$ & $7172(16.7)$ & $5178(23.6)$ & $531(10.9)$ & $424(9.1)$ & $178(8.2)$ & $238(10.3)$ & $254(9.7)$ & $342(8.4)$ \\
\hline $60-69$ & $8754(20.4)$ & $4621(21.0)$ & $1118(23.0)$ & 894 (19.2) & $386(17.7)$ & $463(20.0)$ & $548(21.0)$ & $665(16.3)$ \\
\hline $70-79$ & $10,953(25.5)$ & $3912(17.8)$ & $1626(33.5)$ & $1611(34.5)$ & $675(31.0)$ & $840(36.2)$ & $922(35.3)$ & $1269(31.2)$ \\
\hline $80-89$ & $8880(20.7)$ & $2653(12.1)$ & $1285(26.4)$ & $1392(29.9)$ & $753(34.6)$ & $653(28.2)$ & 703 (26.9) & $1380(33.9)$ \\
\hline$\geq 90$ & $1606(3.7)$ & $579(2.6)$ & $173(3.6)$ & $228(4.9)$ & $134(6.2)$ & $70(3.0)$ & $112(4.3)$ & $304(7.5)$ \\
\hline \multicolumn{9}{|l|}{$\operatorname{Sex}[n(\%)]$} \\
\hline Females & $16,053(37.4)$ & $8371(38.1)$ & $1908(39.3)$ & $1629(34.9)$ & $849(39.0)$ & 785 (33.9) & 764 (29.3) & $1677(41.2)$ \\
\hline $\begin{array}{l}\text { Follow-up days } \\
\text { from hospital } \\
\text { admission } \\
\text { [median (IQR)] }\end{array}$ & $24(10-35)$ & $26(13-36)$ & $23(9-35)$ & $20(8-34)$ & $20(7-33)$ & $21(8-34)$ & $21(8-34)$ & $18(7-33)$ \\
\hline $\begin{array}{l}\text { Admitted to ICU } \\
{[n(\%)]}\end{array}$ & $4392(10.2)$ & $2424(11.0)$ & $502(10.3)$ & $411(8.8)$ & $181(8.3)$ & 279 (12.0) & 307 (11.8) & $261(6.4)$ \\
\hline \multicolumn{9}{|l|}{$\begin{array}{l}\text { No. of hospi- } \\
\text { talisations } \\
\text { within the last } \\
12 \text { months } \\
{[n(\%)]}\end{array}$} \\
\hline 0 & $31,458(73.3)$ & $17,685(80.5)$ & $3530(72.6)$ & 3202 (68.7) & $1311(60.2)$ & $1608(69.4)$ & $1776(68.1)$ & 2185 (53.7) \\
\hline 1 & $6452(15.0)$ & $2722(12.4)$ & $776(16.0)$ & 819 (17.6) & 409 (18.8) & 392 (16.9) & $456(17.5)$ & $826(20.3)$ \\
\hline 2 & $2600(6.1)$ & $868(4.0)$ & $338(7.0)$ & $328(7.0)$ & $201(9.2)$ & $171(7.4)$ & $211(8.1)$ & $460(11.3)$ \\
\hline$\geq 3$ & $2416(5.6)$ & 699 (3.2) & $215(4.4)$ & 314 (6.7) & $257(11.8)$ & $147(6.3)$ & $166(6.4)$ & 597 (14.7) \\
\hline \multicolumn{9}{|l|}{$\begin{array}{l}\text { Charlson index } \\
{[n(\%)]}\end{array}$} \\
\hline 0 & 29,775 (69.4) & $17,979(81.8)$ & 3225 (66.4) & $2716(58.2)$ & $1061(48.7)$ & $1416(61.1)$ & $1534(58.8)$ & $1718(42.2)$ \\
\hline $1-2$ & $10,575(24.6)$ & $3463(15.8)$ & $1373(28.3)$ & $1580(33.9)$ & 819 (37.6) & 724 (31.2) & $861(33.0)$ & $1645(40.4)$ \\
\hline$\geq 3$ & $2576(6.0)$ & $532(2.4)$ & $261(5.4)$ & 367 (7.9) & $298(13.7)$ & $178(7.7)$ & $214(8.2)$ & 705 (17.3) \\
\hline \multicolumn{9}{|l|}{$\begin{array}{l}\text { No. of pharmacy } \\
\text { claims [median } \\
\text { (IQR)] }\end{array}$} \\
\hline $\begin{array}{l}\text { Within the last } \\
12 \text { months }\end{array}$ & $15(3-36)$ & $3(0-13)$ & $27(14-44)$ & $30(17-49)$ & $36(20-56)$ & $36(22-56)$ & $34(20-53)$ & $37(21-59)$ \\
\hline $\begin{array}{l}\text { Within the last } \\
3 \text { months }\end{array}$ & $3(0-9)$ & $0(0-3)$ & $7(3-11)$ & $7(4-12)$ & $8(5-14)$ & $9(5-14)$ & $9(5-13)$ & $9(5-14)$ \\
\hline \multicolumn{9}{|l|}{$\begin{array}{l}\text { Drug use } \\
\text { within the last } \\
12 \text { months } \\
{[n(\%)]}\end{array}$} \\
\hline Anticoagulants & $6743(15.7)$ & $1708(7.8)$ & $937(19.3)$ & 1039 (22.3) & $523(24)$ & $481(20.8)$ & $529(20.3)$ & $1468(36.1)$ \\
\hline $\begin{array}{l}\text { Platelet aggrega- } \\
\text { tion inhibitors }\end{array}$ & 9025 (21.0) & $1649(7.5)$ & 1429 (29.4) & 1659 (35.6) & $826(37.9)$ & 851 (36.7) & 964 (36.9) & $1533(37.7)$ \\
\hline $\begin{array}{l}\text { Lipid-modifying } \\
\text { agents }\end{array}$ & $12,550(29.2)$ & 2763 (12.6) & $2132(43.9)$ & $2244(48.1)$ & $978(44.9)$ & $1182(51.0)$ & $1236(47.4)$ & $1859(45.7)$ \\
\hline $\begin{array}{l}\text { Antiarrhyth- } \\
\text { mics, class } \\
\text { I-III }\end{array}$ & $1924(4.5)$ & 345 (1.6) & $329(6.8)$ & $316(6.8)$ & $148(6.8)$ & 177 (7.6) & $155(5.9)$ & 433 (10.6) \\
\hline
\end{tabular}


Table 1 (continued)

\begin{tabular}{|c|c|c|c|c|c|c|c|c|}
\hline & $\begin{array}{l}\text { All COVID-19 } \\
\text { patients }\end{array}$ & $\begin{array}{l}\text { No anti-HT } \\
\text { use }\end{array}$ & $\mathrm{ARB}$ use & ACEI use & CCB use & $\begin{array}{l}\mathrm{ARB}+\mathrm{CCB} \\
\text { use (fixed } \\
\text { or non-fixed } \\
\text { combination) }\end{array}$ & $\begin{array}{l}\text { ACEI + CCB } \\
\text { use (fixed or } \\
\text { non-fixed com- } \\
\text { bination) }\end{array}$ & $\begin{array}{l}\text { Other anti-HT } \\
\text { use }\end{array}$ \\
\hline $\begin{array}{l}\text { Drugs for peptic } \\
\text { ulcer }\end{array}$ & $16,192(37.7)$ & 4762 (21.7) & 2374 (48.9) & 2395 (51.4) & $1326(60.9)$ & $1233(53.2)$ & $1310(50.2)$ & $2645(65.0)$ \\
\hline $\begin{array}{l}\text { Drugs for } \\
\text { obstructive } \\
\text { airway dis- } \\
\text { eases }\end{array}$ & $6356(14.8)$ & 2337 (10.6) & $927(19.1)$ & 781 (16.7) & 467 (21.4) & $464(20.0)$ & $411(15.8)$ & 919 (22.6) \\
\hline Antibiotics & $17,513(40.8)$ & $7372(33.5)$ & $2280(46.9)$ & 2177 (46.7) & $1119(51.4)$ & $1084(46.8)$ & $1172(44.9)$ & $2190(53.8)$ \\
\hline Anti-HIV drugs & $300(0.7)$ & $136(0.6)$ & $28(0.6)$ & $28(0.6)$ & $24(1.1)$ & $9(0.4)$ & $21(0.8)$ & $48(1.2)$ \\
\hline $\begin{array}{l}\text { Anti-Parkinson } \\
\text { drugs }\end{array}$ & 754 (1.8) & $273(1.2)$ & $88(1.8)$ & $112(2.4)$ & $55(2.5)$ & $38(1.6)$ & $62(2.4)$ & $121(3.0)$ \\
\hline Antiepileptics & $2936(6.8)$ & $1056(4.8)$ & $336(6.9)$ & 401 (8.6) & $215(9.9)$ & $183(7.9)$ & $209(8.0)$ & $508(12.5)$ \\
\hline Antipsychotics & $1737(4.0)$ & $768(3.5)$ & $172(3.5)$ & $217(4.7)$ & $98(4.5)$ & $76(3.3)$ & $94(3.6)$ & $304(7.5)$ \\
\hline Antidepressants & $5339(12.4)$ & 1899 (8.6) & 759 (15.6) & 695 (14.9) & $374(17.2)$ & $360(15.5)$ & 381 (14.6) & $830(20.4)$ \\
\hline \multicolumn{9}{|l|}{$\begin{array}{l}\text { Drug use } \\
\text { within the } \\
\text { last } 3 \text { months } \\
{[n(\%)]}\end{array}$} \\
\hline NSAIDs & $2680(6.2)$ & $993(4.5)$ & $390(8.0)$ & $396(8.5)$ & $159(7.3)$ & $191(8.2)$ & $217(8.3)$ & 307 (7.5) \\
\hline $\begin{array}{l}\text { Corticosteroids } \\
\text { for systemic } \\
\text { use }\end{array}$ & $2358(5.5)$ & $806(3.7)$ & $290(6.0)$ & $336(7.2)$ & $193(8.9)$ & $149(6.4)$ & $170(6.5)$ & $400(9.8)$ \\
\hline $\begin{array}{l}\text { Chloroquine/ } \\
\text { hydroxychlo- } \\
\text { roquine }\end{array}$ & $222(0.5)$ & $69(0.3)$ & $42(0.9)$ & $35(0.8)$ & $13(0.6)$ & $14(0.6)$ & $16(0.6)$ & $28(0.7)$ \\
\hline $\begin{array}{l}\text { Immunosup- } \\
\text { pressants }\end{array}$ & 695 (1.6) & $234(1.1)$ & $74(1.5)$ & $93(2.0)$ & 84 (3.9) & 43 (1.9) & $53(2.0)$ & $106(2.6)$ \\
\hline \multicolumn{9}{|l|}{$\begin{array}{l}\text { Comorbidities } \\
{[n(\%)]}\end{array}$} \\
\hline $\begin{array}{l}\text { Ischaemic heart } \\
\text { disease }\end{array}$ & $4436(10.3)$ & $710(3.2)$ & $632(13)$ & 903 (19.4) & $378(17.4)$ & $343(14.8)$ & 432 (16.6) & $994(24.4)$ \\
\hline $\begin{array}{l}\text { Atrial fibrilla- } \\
\text { tion }\end{array}$ & $2899(6.8)$ & $550(2.5)$ & $379(7.8)$ & $485(10.4)$ & $221(10.1)$ & $188(8.1)$ & $229(8.8)$ & $819(20.1)$ \\
\hline Heart failure & $2684(6.3)$ & $447(2.0)$ & 335 (6.9) & 498 (10.7) & $229(10.5)$ & $136(5.9)$ & $183(7.0)$ & $831(20.4)$ \\
\hline Hypertension & $5610(13.1)$ & $1097(5.0)$ & 897 (18.5) & 878 (18.8) & $552(25.3)$ & $535(23.1)$ & $626(24.0)$ & $960(23.6)$ \\
\hline $\begin{array}{l}\text { Cerebrovascular } \\
\text { diseases }\end{array}$ & $3441(8.0)$ & $1041(4.7)$ & $387(8.0)$ & $474(10.2)$ & 305 (14.0) & 247 (10.7) & $330(12.6)$ & $623(15.3)$ \\
\hline $\begin{array}{l}\text { Diabetes mel- } \\
\text { litus }\end{array}$ & $7710(18.0)$ & $1946(8.9)$ & $1234(25.4)$ & $1206(25.9)$ & 608 (27.9) & $718(31.0)$ & $818(31.4)$ & $1082(26.6)$ \\
\hline Liver disease & 701 (1.6) & $239(1.1)$ & $75(1.5)$ & $81(1.7)$ & $55(2.5)$ & $41(1.8)$ & $37(1.4)$ & $170(4.2)$ \\
\hline Dementia & $1014(2.4)$ & $444(2.0)$ & $90(1.9)$ & $126(2.7)$ & $70(3.2)$ & $46(2.0)$ & $50(1.9)$ & $183(4.5)$ \\
\hline $\begin{array}{l}\text { Pneumonia and } \\
\text { influenza }\end{array}$ & $2737(6.4)$ & $885(4.0)$ & $274(5.6)$ & $353(7.6)$ & $242(11.1)$ & $134(5.8)$ & 197 (7.6) & $634(15.6)$ \\
\hline Renal failure & $1046(2.4)$ & $255(1.2)$ & $97(2.0)$ & $106(2.3)$ & $158(7.3)$ & $74(3.2)$ & $74(2.8)$ & $270(6.6)$ \\
\hline COPD & $1521(3.5)$ & $389(1.8)$ & $178(3.7)$ & $210(4.5)$ & $162(7.4)$ & $108(4.7)$ & $87(3.3)$ & $376(9.2)$ \\
\hline Cancer & $6446(15.0)$ & $2520(11.5)$ & $780(16.1)$ & 875 (18.8) & $479(22.0)$ & 409 (17.6) & $455(17.4)$ & 883 (21.7) \\
\hline $\begin{array}{c}\text { Rheumatic } \\
\text { diseases }\end{array}$ & $375(0.9)$ & $107(0.5)$ & $48(1.0)$ & $51(1.1)$ & 42 (1.9) & $23(1.0)$ & $18(0.7)$ & $81(2.0)$ \\
\hline
\end{tabular}

$A C E I$ angiotensin-converting enzyme inhibitor, $A R B$ angiotensin receptor blocker, $C C B$ calcium channel blocker, $C O P D$ chronic obstructive pulmonary disease, anti-HT antihypertensive drugs (includes $\alpha 2$-adrenergic agonist, diuretics and $\beta$-blockers), ICU intensive care unit, IQR interquartile range, $H I V$ human immunodeficiency virus, NSAIDs non-steroidal anti-inflammatory drugs

${ }^{a}$ We removed 257 patients from the analysis who were concomitantly treated with ARBs and ACEIs ( \pm CCBs or other antihypertensive drugs). The mortality rate of these patients was comparable with that of all other antihypertensive categories 
Table 2 Risk factors of all-cause mortality in COVID-19 hospitalised patients, at univariate analysis

\begin{tabular}{|c|c|c|c|}
\hline Covariate & $\begin{array}{l}\text { No. of COVID-19 patients } \\
{[n=42,926]}\end{array}$ & $\begin{array}{l}\text { No. of deaths }(\%) \\
{[n=11,205(26.1)]}\end{array}$ & Unadjusted HR (95\% CI) \\
\hline \multicolumn{4}{|l|}{ Centre } \\
\hline Lombardy & 38,715 & $10,569(27.3)$ & Ref. \\
\hline Reggio Emilia & 982 & $197(20.1)$ & $0.98(0.86-1.13)$ \\
\hline Veneto & 3229 & $439(13.6)$ & $0.66(0.60-0.72)$ \\
\hline \multicolumn{4}{|l|}{ Age, years } \\
\hline $18-49$ & 5561 & $141(2.5)$ & Ref. \\
\hline $50-59$ & 7172 & $451(6.3)$ & $2.49(2.06-3.01)$ \\
\hline $60-69$ & 8754 & $1484(17.0)$ & $7.04(5.92-8.37)$ \\
\hline $70-79$ & 10,953 & $3867(35.3)$ & $16.8(14.2-19.8)$ \\
\hline $80-89$ & 8880 & $4343(48.9)$ & $27.6(23.3-32.6)$ \\
\hline$\geq 90$ & 1606 & $919(57.2)$ & $37.5(31.4-44.8)$ \\
\hline \multicolumn{4}{|l|}{ Sex } \\
\hline Male & 26,873 & $7662(28.5)$ & Ref. \\
\hline Female & 16,053 & $3543(22.1)$ & $0.77(0.74-0.80)$ \\
\hline \multicolumn{4}{|c|}{ No. of hospitalisations within the last 12 months } \\
\hline 0 & 31,458 & $7181(22.8)$ & Ref. \\
\hline 1 & 6452 & $2013(31.2)$ & $1.45(1.38-1.52)$ \\
\hline 2 & 2600 & $1015(39.0)$ & $1.93(1.80-2.06)$ \\
\hline$\geq 3$ & 2416 & $996(41.2)$ & $2.05(1.92-2.19)$ \\
\hline \multicolumn{4}{|l|}{ Charlson index } \\
\hline 0 & 29,775 & $5805(19.5)$ & Ref. \\
\hline $1-2$ & 10,575 & $4018(38.0)$ & $2.24(2.15-2.33)$ \\
\hline$\geq 3$ & 2576 & $1382(53.6)$ & $3.65(3.44-3.87)$ \\
\hline Drug use within the last 12 months & & & vs. non-use \\
\hline Anticoagulants & 6743 & $2632(39.0)$ & $1.84(1.76-1.92)$ \\
\hline Platelet aggregation inhibitors & 9025 & $3775(41.8)$ & $2.22(2.14-2.31)$ \\
\hline Lipid-modifying agents & 12,550 & $4516(36.0)$ & $1.78(1.72-1.85)$ \\
\hline Antiarrhythmics, class I and III & 1924 & $800(41.6)$ & $1.82(1.70-1.96)$ \\
\hline Drugs for peptic ulcer & 16,192 & $5738(35.4)$ & $1.91(1.84-1.98)$ \\
\hline Drugs for obstructive airway diseases & 6356 & $2052(32.3)$ & $1.33(1.27-1.40)$ \\
\hline Antibiotics & 17,513 & $5066(28.9)$ & $1.22(1.17-1.27)$ \\
\hline Anti-HIV drugs & 300 & $95(31.7)$ & $1.22(1.00-1.50)$ \\
\hline Anti-Parkinson drugs & 754 & $357(47.3)$ & $2.20(1.98-2.44)$ \\
\hline Antiepileptics & 2936 & $1063(36.2)$ & $1.54(1.45-1.64)$ \\
\hline Antipsychotics & 1737 & $750(43.2)$ & $2.01(1.86-2.16)$ \\
\hline Antidepressants & 5339 & $1935(36.2)$ & $1.62(1.54-1.70)$ \\
\hline Drug use within the last 3 months & & & vs. non-use \\
\hline NSAIDs & 2680 & $781(29.1)$ & $1.15(1.07-1.23)$ \\
\hline Corticosteroids for systemic use & 2358 & $847(35.9)$ & $1.51(1.41-1.62)$ \\
\hline Chloroquine/hydroxychloroquine & 222 & $78(35.1)$ & $1.44(1.16-1.80)$ \\
\hline Immunosuppressants & 695 & $183(26.3)$ & $1.01(0.87-1.16)$ \\
\hline Comorbidities & & & vs. absence of the disease \\
\hline Ischaemic heart disease & 4436 & $2013(45.4)$ & $2.22(2.12-2.33)$ \\
\hline Atrial fibrillation & 2899 & $1323(45.6)$ & $2.19(2.07-2.32)$ \\
\hline Heart failure & 2684 & $1416(52.8)$ & $2.74(2.59-2.90)$ \\
\hline Hypertension & 5610 & $2396(42.7)$ & $2.11(2.02-2.21)$ \\
\hline Cerebrovascular diseases & 3441 & $1554(45.2)$ & $2.23(2.12-2.36)$ \\
\hline Diabetes mellitus & 7710 & $3041(39.4)$ & $1.92(1.84-2.00)$ \\
\hline Pneumonia and influenza & 2737 & $1313(48.0)$ & $2.34(2.21-2.48))$ \\
\hline
\end{tabular}


Table 2 (continued)

\begin{tabular}{llll}
\hline Covariate & $\begin{array}{l}\text { No. of COVID-19 patients } \\
{[n=42,926]}\end{array}$ & $\begin{array}{l}\text { No. of deaths (\%) } \\
{[n=11,205(26.1)]}\end{array}$ & Unadjusted HR (95\% CI) \\
\hline Liver disease & 701 & $286(40.8)$ & $1.73(1.54-1.94)$ \\
Dementia & 1014 & $535(52.8)$ & $2.76(2.53-3.01)$ \\
Renal failure & 1046 & $498(47.6)$ & $2.24(2.05-2.45)$ \\
COPD & 1521 & $699(46.0)$ & $2.10(1.94-2.27)$ \\
Cancer & 6446 & $2344(36.4)$ & $1.63(1.56-1.70)$ \\
Rheumatic diseases & 375 & $159(42.4)$ & $1.96(1.67-2.29)$ \\
\hline
\end{tabular}

$H R$ hazard ratio, $C I$ confidence interval, $C O P D$ chronic obstructive pulmonary disease, $H I V$ human immunodeficiency virus, NSAID non-steroidal anti-inflammatory drug, Ref. reference group

Table 3 Unadjusted and adjusted hazard ratio of all-cause mortality in hospitalised COVID-19 patients in relation to antihypertensive exposure within the last 3 months of observation

\begin{tabular}{|c|c|c|c|c|}
\hline & $\begin{array}{l}\text { No of deaths (\% of } \\
\text { total users) }\end{array}$ & No. of total users & Unadjusted HR (95\% CI) & Adjusted $\mathrm{HR}^{\mathrm{a}}(95 \% \mathrm{CI})$ \\
\hline $\mathrm{ARBs}$ & $1540(31.7)$ & 4859 & $0.79(0.72-0.86)$ & $0.98(0.89-1.06)$ \\
\hline ACEIs & $1606(34.4)$ & 4663 & $0.89(0.81-0.96)$ & $0.97(0.89-1.06)$ \\
\hline Other anti-HT drugs & $1634(40.2)$ & 4068 & $1.08(1.00-1.18)$ & $1.05(0.96-1.14)$ \\
\hline $\mathrm{ARBs}+\mathrm{CCBs}^{\mathrm{b}}$ & $2399(33.4)$ & 7177 & $0.84(0.77-0.91)$ & $1.02(0.94-1.11)$ \\
\hline $\mathrm{ACEIs}+\mathrm{CCBs}^{\mathrm{c}}$ & $2496(34.3)$ & 7272 & $0.88(0.82-0.96)$ & $0.97(0.90-1.05)$ \\
\hline $\mathrm{CCBs}$ & $822(37.7)$ & 2178 & Reference category & Reference category \\
\hline $\mathrm{ARBs}$ & $1540(31.7)$ & 4859 & $1.99(1.87-2.11)$ & $1.12(1.05-1.20)$ \\
\hline ACEIs & $1606(34.4)$ & 4663 & $2.24(2.11-2.37)$ & $1.10(1.03-1.17)$ \\
\hline $\mathrm{CCBs}$ & $822(37.7)$ & 2178 & $2.52(2.34-2.72)$ & $1.11(1.03-1.21)$ \\
\hline $\mathrm{ARBs}+\mathrm{CCBs}^{\mathrm{d}}$ & $859(37.1)$ & 2318 & $2.40(2.23-2.58)$ & $1.29(1.19-1.40)$ \\
\hline $\mathrm{ACEIs}+\mathrm{CCBs}^{\mathrm{e}}$ & $890(34.1)$ & 2609 & $2.21(2.05-2.37)$ & $1.13(1.04-1.22)$ \\
\hline Other anti-HT drugs & $1634(40.2)$ & 4068 & $2.73(2.58-2.90)$ & $1.17(1.09-1.25)$ \\
\hline Non-use of any anti-HT drug & $3766(17.1)$ & 21,974 & Reference category & Reference category \\
\hline
\end{tabular}

ACEIs ACE inhibitors, ARBs angiotensin receptor blockers, $C C B s$ calcium channel blockers, $C I$ confidence interval, $C O P D$ chronic obstructive pulmonary disease, anti-HT antihypertensive drugs ( $\alpha 2$-adrenergic agonist, diuretics, and $\beta$-blockers), $H R$ hazard ratio, $H I V$ human immunodeficiency virus, NSAIDs non-steroidal anti-inflammatory drugs

${ }^{a}$ Cox proportional regression model (stepwise forward based on Akaike's information criterion) adjusted for the following eligible variables: centre, age, sex, Charlson index, number of drug dispensings, drugs for peptic ulcer, anticoagulants, antiplatelet drugs, lipid-modifying agents, antiarrhythmic drugs, drugs for obstructive airway diseases, antibiotics, anti-HIV drugs, anti-Parkinson drugs, antiepileptic drugs, antipsychotics, antidepressants, NSAIDS, corticosteroids, chloroquine/hydroxychloroquine, immunosuppressants, pneumonia and influenza, ischaemic heart disease, atrial fibrillation, heart failure, hypertension, cerebrovascular diseases, diabetes mellitus, liver disease, dementia, renal failure, COPD, cancer, and rheumatic diseases

${ }^{\mathrm{b}} \mathrm{ARBs}$ as monotherapy or fixed combination with other HT drugs, except for ACEIs and CCBs or ARBs + CCBs as fixed combination

${ }^{\mathrm{c}} \mathrm{ACEI}$ as monotherapy or fixed combination with other HT drugs, except for ARBs and CCBs or ACEIs + CCBs as fixed combination

${ }^{\mathrm{d}} \mathrm{ARBs}+\mathrm{CCBs}$ as fixed combination

${ }^{\mathrm{e}} \mathrm{ACEI}+\mathrm{CCBs}$ as fixed combination

for users of ACEIs (HR 1.10, 95\% CI 1.03-1.17), ARBs (HR 1.12, 95\% CI 1.05-1.20) and CCBs (HR 1.11, 95\% CI 1.03-1.21). Among hypertensive patients only, this effect disappeared (Fig. 1). The main finding that there is no significantly increased risk of death with ACEI/ARB treatment compared with CCB treatment was confirmed in other sensitivity analyses, including the PS-matched analysis, which included 2031 ARB users (41.9\% of all ARB users in the cohort) matched to $2031 \mathrm{CCB}$ users (93.2\% of all CCB users in the cohort), and 2094 ACEI users (45.1\% of all ACEI users in the cohort) matched to 2094 CCB users $(96.1 \%$ of all CCB users in the cohort) (Fig. 1). Results for in-hospital death as the outcome $(n=9878)$ were very similar to death defined for the main analysis, with no significant risk for ARBs or ACEIs compared with CCBs (HR 0.99, 95\% CI 0.90-1.07; and HR 0.98, 95\% CI 0.90-1.07, respectively). 


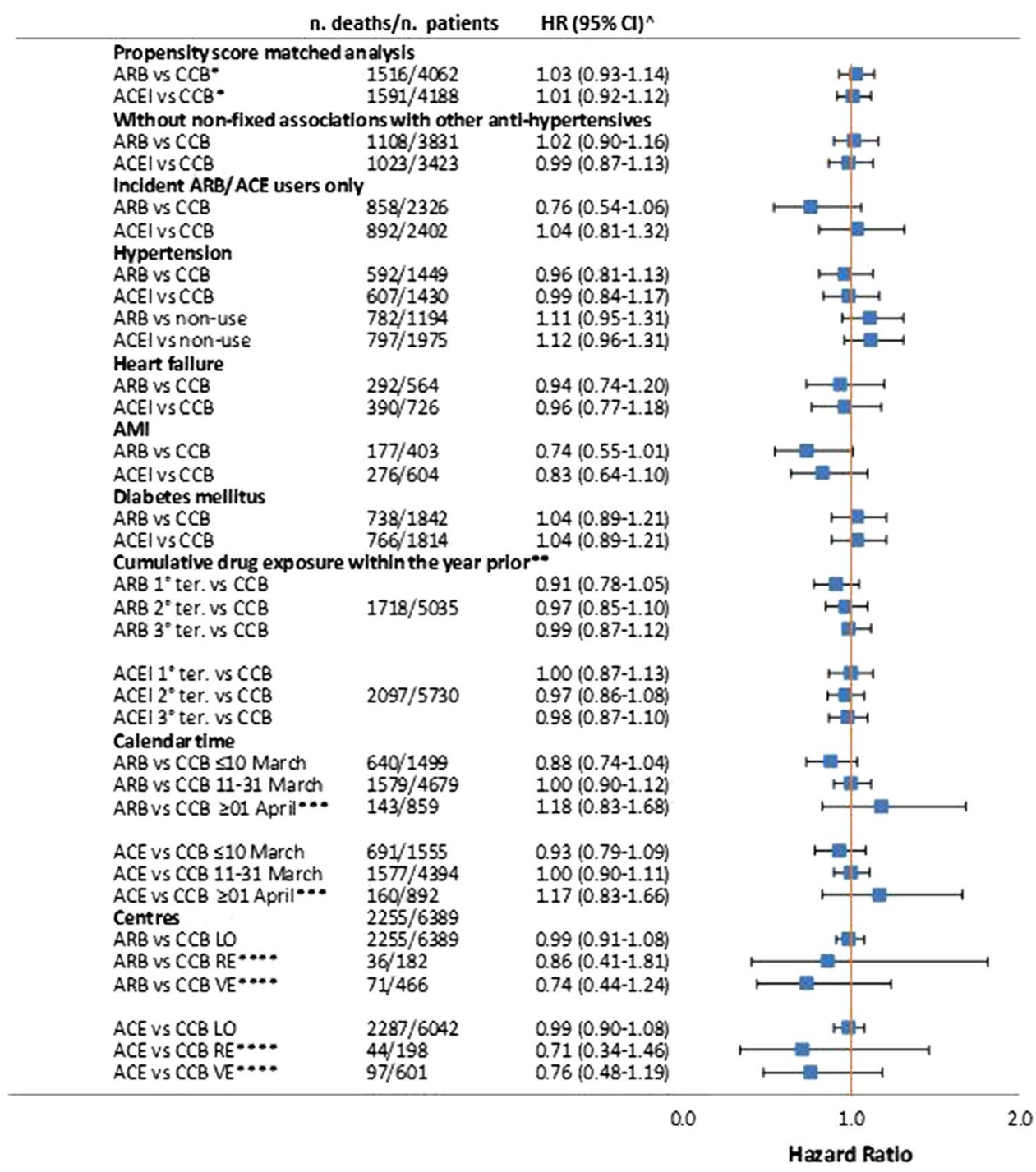

Fig. 1 Sensitivity and subgroup analyses of all-cause mortality risk in hospitalised COVID-19 patients in association with ACEIs/ARBs vs. CCBs (or non-use). AMI acute myocardial infarction, ACEIs angiotensin-converting enzyme inhibitors, $A R B s$ angiotensin receptor blocker, $C C B$ s calcium channel blockers, $C I$ confidence interval, $A H$ antihypertensive drugs ( $\alpha 2$-adrenergic agonist, diuretics, and $\beta$-blockers, NSAIDs non-steroidal anti-inflammatory drugs, COPD chronic obstructive pulmonary disease, $L O$ Lombardy region, HIV human immunodeficiency virus, $H R$ hazard ratio, $R E$ Reggio Emilia LHU, $V E$ Veneto region, $D D D s$ defined daily doses. ${ }^{\wedge}$ Cox proportional regression model (stepwise forward based on Akaike's information criterion) adjusted for the following eligible variables: centre, age, sex, Charlson index, number of drug dispensings, drugs for peptic ulcer, anticoagulants, antiplatelet drugs, lipid-modifying agents,

Findings regarding in-hospital death were also similar to the main analysis for ARBs and ACEIs in comparison with the non-use of antihypertensive drugs (HR 1.13, 95\% CI 1.06-1.21; and HR 1.12, 95\% 1.04-1.20, respectively). There was no trend in mortality risk on increasing cumulative exposure in comparison with CCBs, either for ACEIs antiarrhythmics, drugs for obstructive airway diseases, antibiotics, anti-HIV drugs, anti-Parkinson drugs, antiepileptics, antipsychotics, antidepressants, NSAIDS, corticosteroids, chloroquine/hydroxychloroquine, immunosuppressants, pneumonia and influenza, ischaemic heart disease, atrial fibrillation, heart failure, hypertension, cerebrovascular diseases, diabetes mellitus, liver disease, dementia, renal failure, COPD, cancer, and rheumatic diseases. *Propensity-score matched Cox regression model. **ARB: $1^{\circ}$ tertile: upper tertile of number of DDDs in the last year: $\angle 224 ; 2^{\circ}$ tertile: intermediate tertile: $224-419 ; 3^{\circ}$ tertile: low tertile: $\geq 420$; ACEI: $1^{\circ}$ tertile: upper tertile $<280 ; 2^{\circ}$ tertile: intermediate tertile: $280-615 ; 3^{\circ}$ tertile: low tertile: $\geq 616$. $* * *$ Only the Lombardy region. $* * * *$ Cox regression model adjusted by propensity score

or ARBs. Interestingly, there was a non-significant trend for calendar time, where, both for ACEIs and ARBs, there was a non-significant higher risk of death after 11 April 2020. Stratification by catchment area resulted in findings that were likely underpowered for Veneto and Reggio Emilia, although these results are also in line with the main analysis. 


\section{Discussion}

The present study is the largest to date to investigate the safety of ACEIs and ARBs in persons with COVID-19. The main finding of this study was that prior exposure to ACEIs/ ARBs among new users of these drugs neither worsened nor improved the prognosis of hospitalised COVID-19 patients in terms of all-cause mortality compared with CCBs. The sample size of the study allowed us to conduct several supporting and sensitivity analyses. We observed no increased risk of death among ACEI or ARB users having the highest drug cumulative exposure (upper tertile of DDDs) within 1 year prior to the ID, compared with CCB use. This finding was confirmed in several supporting and sensitivity analyses, using the PS matching approach or changing exposure and outcome definitions.

When considering non-use as a comparator, the $2 / 2.5$ fold increased risk of death with all antihypertensive drugs in unadjusted analyses almost completely disappeared after controlling for confounders, yielding a marginally significant $10-15 \%$ increased mortality risk, likely attributable to residual confounding by indication. Accordingly, in hypertensive patients specifically, no statistically significant difference in risk for ACEI/ARB use versus non-use of antihypertensives was observed. This finding is in line with three small Chinese retrospective studies demonstrating that inhospital exposure to ACEIs/ARBs in hypertensive COVID19 patients was not associated with increased mortality [14, 24]. Larger studies have also shown a lack of association between these drug classes and severe COVID-19, as measured by outcomes such as intensive care or death [11,26].

The studies reporting a potentially protective effect were either not published in peer-reviewed scientific journals [14, 27], were not full research papers but letters with limited detail [28], or were retracted [29]. While previous observational studies provided preliminary evidence that there is no additional risk of mortality with RAAS inhibitors compared with other antihypertensive drugs in COVID-19 patients, estimates from this large-scale study, based on extensive adjustment for potential confounders and numerous sensitivity analyses, confirm that there is no increase in risk. In addition, we showed that there is no protective effect of ACEIs/ ARBs, as previously reported in a meta-analysis of observational studies [18] that were mainly conducted through retrospective assessment of small cohorts of hospitalised patients with COVID-19. The present study is based on a much larger study population, more extensive adjustment for confounding and a larger battery of sensitivity analyses.

This study has several strengths. First, we performed the study using a large multiple database network with quasi real-time data access for over 40,000 COVID-19 hospitalised patients from three regions, each of which was heavily affected by SARS-CoV-2 infection. The data used in the present study were collected while the pandemic was at its peak, increasing the significance of our findings. While an observational study set in Lombardy has already been published [11], the present study has over 1 month of additional data, with a population size that is approximately seven times as large. The estimated overlap between our hospitalised population and that of Mancia et al. is approximately $6 \%$, with the total number of ACEI/ARB users being around 720 [11]. Moreover, the study design by Mancia et al. was not intended to evaluate how RAAS inhibitors could affect the prognosis among persons with COVID-19, and therefore may not be very informative in this regard. Second, the use of the COVID-19 patient registry, which is maintained by the Italian NHS and updated daily, leverages accurately collected data on patients testing positive for SARS-CoV-2 RNA by PCR on nasopharyngeal/throat swabs. Third, this is the largest cohort of COVID-19 patients, at least in Europe, that has been analysed at a population-based level to date. This large sample size allowed us to perform a number of sensitivity/subgroup analyses, thus increasing the robustness of study findings. A variety of approaches were used to control for confounding, including stratification by comorbidity and cumulative exposure, as well as the use of PSs. We were able to adjust for a very large number of potential confounders by leveraging Italian NHS claims databases that have been used widely, by linking them at the individual patient level to the COVID-19 surveillance registry. Regarding the exposure, in the present study we have distinguished between ACEI and ARB use as monotherapy or combination therapy, which has not always been done in previous studies $[14,23,24,30]$. We selected a comparator, CCB use, which is a therapeutic alternative to ACEI/ARB use, making our findings relevant to decision making in clinical practice. This has also not always been done in previous studies [14, 23, $24,30]$. Finally, to our knowledge, the present study is the only study that has investigated the dose-effect response on the risks associated with ACEI/ARB use. Dose-effect analyses are essential to demonstrate the biological plausibility of a hypothesised drug-related risk. A different outcome definition was also applied, i.e. in-hospital mortality, in addition to the primary outcome definition, i.e. all-cause mortality in any setting. This not only further confirms the main findings but also shows the lack of competing risks.

Nevertheless, some limitations warrant caution. We had access to pharmacy claims of COVID-19 patients up to 29 February 2020, 31 December 2019 and 30 November 2019 in Reggio Emilia, Lombardy and Veneto, respectively. As such, we may have misclassified exposure, especially in Lombardy and Veneto, if antihypertensive users withdrew/ switched therapies due to concerns about SARS-COV-2 infection in the period immediately preceding COVID-19 
hospitalisation. However, in the trimester prior to COVID19 hospitalisation in Reggio Emilia, only $1.5 \%$ and $1.4 \%$ of ACEI and ARB users, respectively, switched to CCBs. We have no reason to believe that this trend was different in the other two Northern Italian catchment areas considered. COVID-19 patients with the lowest cumulative exposure to antihypertensive drugs in the past year (as a proxy of low adherence), who may be more likely to withdraw therapy, were not associated with higher death risk, compared with CCBs. We did not have information on risk factors for death in COVID-19 patients such as obesity and smoking; however, we adjusted the analysis for comorbidities strictly correlated to these variables (e.g. diabetes mellitus and chronic obstructive pulmonary disease), thus potentially accounting for their confounding effect. We explored the exposure status only in an outpatient setting, and we do not know if antihypertensive drugs were continued during hospital stay. Finally, some comorbidities that are not likely to be the cause of hospitalisation, especially chronic conditions such as hypertension and diabetes, may be underestimated as they were mainly identified from hospital discharge diagnoses. Another limitation is that there may be potential bias in our cohort of hospitalised patients as the criteria for hospital admission are not defined and may depend on several factors, including severity, availability of hospital beds, etc., that are likely to vary from hospital to hospital. Nevertheless, a case-control study conducted in Italy has shown that there is no difference between ACEI or ARB use and the risk of hospitalisation among COVID-19 patients [11].

\section{Conclusion}

This large Italian population-based cohort study demonstrated that prior exposure to ACEIs and ARBs is not associated with either an increased or decreased risk of all-cause mortality in hospitalised COVID-19, in comparison with CCBs. This finding strengthens recommendations from international regulatory drug agencies and scientific societies about not withdrawing ACEI/ARB therapies or switching to other antihypertensive drugs for the prevention of COVID-19-related death. Furthermore, this study shows that real-world evidence generation during healthcare emergencies may rapidly confirm or refute hypotheses derived from in vitro studies.

Acknowledgements The members of ITA COVID-19: RAAS Inhibitor Group are: Istituto Superiore di Sanità, Rome, Italy: Stefania Spila Alegiani, Francesca Menniti Ippolito, Roberto Da Cas, Marco Massari, Giuseppe Traversa, Graziano Onder; Department of Biomedical and Dental Sciences and Morphofunctional Imaging, University of Messina, Messina, Italy, and Italian Society of Pharmacology, Milan, Italy: Gianluca Trifirò, Janet Sultana, Valentina Ientile, Salvatore
Crisafulli; Azienda Unità Sanitaria Locale - IRCCS, Reggio Emilia, Italy: Paolo Giorgi Rossi, Roberto Grilli, Giulio Formoso, Massimiliano Marino, Massimo Vicentini; Epidemiology Observatory, Department of Health, Lombardy Region, Milan, Italy: Olivia Leoni, Monica Ludergnani, Danilo Cereda; Azienda Zero, Veneto Region, Padova, Italy: Eliana Ferroni, Stefano Guzzinati, Emanuela Bovo, Manuel Zorzi; Aulss 3 Serenissima, Regional Centre for Biomarkers, Venice, Italy: Massimo Gion.

\section{Declarations}

Funding No funding was received for the conduct of this study.

Conflict of interest Marco Massari, Roberto Da Cas, Francesca Menniti Ippolito, Janet Sultana, Salvatore Crisafulli, Paolo Giorgi Rossi, Massimiliamo Marino, Manuel Zorzi, Emanuela Bovo, Olivia Leoni, Monica Ludergnani and Stefania Spila Alegiani have no conflicts of interest to disclose. Gianluca Trifirò reports grants from Novartis, from the Italian Drug Agency, during the conduct of the study; has participated in advisory boards within the last 5 years on topics not related to this article and organised by Sandoz, Hospira, Sanofi, Biogen, Ipsen, and Shire; and is a consultant for Otsuka. He is the principal investigator of observational studies funded by several pharmaceutical companies (e.g. Amgen, AstraZeneca, Daiichi Sankyo, IBSA) to the University of Messina, as well as thescientific coordinator of the Master's programme 'Pharmacovigilance, Pharmacoepidemiology and Pharmacoeconomics: Real-World Data Evaluations' at the University of Messina, which receives unconditional funding from several pharmaceutical companies.

Ethics Approval This study was approved by the Ethics Committee of the Italian National Institute of Health.

Consent to Participate Not applicable.

Consent for Publication Not applicable.

Availability of Data and Material The dataset generated for the present study is not available for sharing.

Code Availability Not applicable.

Author contributions GT, MarM and SSA conceived the idea for this paper. MarM and SSA conducted the statistical data analysis. GT, MarM, RDC, FMI, JS, SC, PGR, MasM, MZ, EB, OL, ML and SSA interpreted the results, and wrote and revised the paper.

\section{References}

1. European Centre for Disease Prevention and Control. Latest situation update, epidemiological curve and global distribution. 2020. https://www.ecdc.europa.eu/en. Accessed 7 July 2020.

2. Italian Ministry of Health. COVID-19-Situation in Italy. 2020. https://www.salute.gov.it/portale/nuovocoronavirus/detta glioContenutiNuovoCoronavirus.jsp?area $=$ nuovoCoronaviru s\&id=5351\&lingua $=$ italiano\&menu=vuoto. Accessed 7 July 2020.

3. Gurwitz D. Angiotensin receptor blockers as tentative SARSCoV-2 therapeutics. Drug Dev Res. 2020;81(5):537-40.

4. Zheng Y-Y, Ma Y-T, Zhang J-Y, Xie X. COVID-19 and the cardiovascular system. Nat Rev Cardiol. 2020;17(5):259-60.

5. Watkins J. Preventing a covid-19 pandemic. BMJ. 2020;368:m810. 
6. Hoffmann M, Kleine-Weber H, Schroeder S, Krüger N, et al. SARS-CoV-2 cell entry depends on ACE2 and TMPRSS2 and is blocked by a clinically proven protease inhibitor. Cell. 2020;181(2):271-280.e8.

7. Ferrario CM, Jessup J, Chappell MC, Averill DB, et al. Effect of angiotensin-converting enzyme inhibition and angiotensin II receptor blockers on cardiac angiotensin-converting enzyme 2 . Circulation. 2005;111(20):2605-10.

8. Ferrario CM, Jessup J, Gallagher PE, Averill DB, et al. Effects of renin-angiotensin system blockade on renal angiotensin-(1-7) forming enzymes and receptors. Kidney Int. 2005;68(5):2189-96.

9. Kuba K, Imai Y, Rao S, Gao H, et al. A crucial role of angiotensin converting enzyme 2 (ACE2) in SARS coronavirus-induced lung injury. Nat Med. 2005;11:875-9.

10. Imai Y, Kuba K, Rao S, Huan Y, et al. Angiotensin-converting enzyme 2 protects from severe acute lung failure. Nature. 2005;436:112-6.

11. Mancia G, Rea F, Ludergnani M, Apolone G, et al. Renin-angiotensin-aldosterone system blockers and the risk of Covid-19. N Engl J Med. 2020;382(25):2431-40.

12. de Abajo FJ, Rodríguez-Martín S, Lerma V, Mejía-Abril G, et al. Use of renin-angiotensin-aldosterone system inhibitors and risk of COVID-19 requiring admission to hospital: a case-population study. Lancet. 2020;395:1705-14.

13. Selçuk M, Çınar T, Keskin M, Çiçek V, et al. Is the use of ACE inb/ARBs associated with higher in-hospital mortality in Covid19 pneumonia patients? Clin Exp Hypertens. 2020;42(8):738-42.

14. Li J, Wang X, Chen J, Zhang H, et al. Association of renin-angiotensin system inhibitors with severity or risk of death in patients with hypertension hospitalized for coronavirus disease 2019 (COVID-19) infection in Wuhan, China. JAMA Cardiol. 2020;5(7):1-6.

15. Yang G, Tan Z, Zhou L, Yang M, et al. Effects of angiotensin II receptor blockers and ACE (angiotensin-converting enzyme) inhibitors on virus infection, inflammatory status, and clinical outcomes in patients with COVID-19 and hypertension. Hypertension. 2020;76:51-8.

16. Feng Y, Ling Y, Bai T, Xie Y, et al. COVID-19 with different severities: a multicenter study of clinical features. Am J Respir Crit Care Med. 2020;201:1380-8.

17. Meng J, Xiao G, Zhang J, Xing H, et al. Renin-angiotensin system inhibitors improve the clinical outcomes of COVID-19 patients with hypertension. Emerg Microbes Infect. 2020;9(1):757-60.

18. Pirola CJ, Sookoian S. Estimation of renin-angiotensin-aldosterone-system (RAAS)-inhibitor effect on COVID-19 outcome: a meta-analysis. J Infect. 2020;81(2):276-81.
19. Zhang X, Yu J, Pan L, Jian HY. ACEI/ARB use and risk of infection or severity or mortality of COVID-19: a systematic review and meta-analysis. Pharmacol Res. 2020;158:104927.

20. Rubin EJ, Mehra MR, et al. Expression of concern: cardiovascular disease, drug therapy, and mortality in covid-19. N Engl J Med. 2020;382:2464.

21. Fang L, Karakiulakis G, Roth M. Are patients with hypertension and diabetes mellitus at increased risk for COVID-19 infection? Lancet Respir Med. 2020;2600:30116.

22. Fosbø1 EL, Butt JH, Østergaard L, Andersson C, et al. Association of angiotensin-converting enzyme inhibitor or angiotensin receptor blocker use with COVID-19 diagnosis and mortality. JAMA. 2020;324(2):168-77.

23. Gao C, Cai Y, Zhang K, Zhou L, et al. Association of hypertension and antihypertensive treatment with COVID-19 mortality: a retrospective observational study. Eur Heart J. 2020;41(22):2058-66.

24. Zhang P, Zhu L, Cai J, Lei F, et al. Association of inpatient use of angiotensin converting enzyme inhibitors and angiotensin II receptor blockers with mortality among patients with hypertension hospitalized With COVID-19. Circ Res. 2020;126(12):1671-81.

25. Trifirò G, Gini R, Barone-Adesi F, Beghi E, et al. The role of european healthcare databases for post-marketing drug effectiveness, safety and value evaluation: where does Italy stand? Drug Saf. 2019;42:347-63.

26. Reynolds HR, Adhikari S, Pulgarin C, Troxel AB, et al. Reninangiotensin-aldosterone system inhibitors and risk of covid-19. N Engl J Med. 2020;382(25):2441-8.

27. Andrew IP, Parikh K, Parrillo J, Mathura S, Hansen E, Sawczuk I, Goldberg S. Hypertension and renin-angiotensin-aldosterone system inhibitors in patients with Covid-19. MedRxiv. 2020. https ://doi.org/10.1101/2020.04.24.20077388.

28. Conversano A, Melillo F, Napolano A, Evgeny F, et al. RAAs inhibitors and outcome in patients with SARS-CoV-2 pneumonia. A case series study. Hypertension. 2020;76(2):e10-e1212.

29. Mehra MR, Desai SS, Kuy S, Henry TD, et al. Cardiovascular disease, drug therapy, and mortality in covid-19. N Engl J Med. 2020;382(25):e102.

30. Tedeschi S, Giannella M, Bartoletti M, Trapani F, et al. Clinical impact of renin-angiotensin system inhibitors on in-hospital mortality of patients with hypertension hospitalized for COVID-19. Clin Infect Dis. 2020;71(15):899-901.

\section{Affiliations}

\section{Gianluca Trifiròn ${ }^{1,2}$ - Marco Massari ${ }^{3} \cdot{\text { Roberto Da } \text { Cas }^{3} \cdot \text { Francesca Menniti Ippolito }^{3} \text {. Janet Sultana }}^{1,2}$. Salvatore Crisafulli ${ }^{1,2} \cdot$ Paolo Giorgi Rossi ${ }^{4} \cdot$ Massimiliano Marino ${ }^{4} \cdot$ Manuel Zorzi $^{5} \cdot$ Emanuela Bovo $^{5}$. Olivia Leoni ${ }^{6}$. Monica Ludergnani ${ }^{6}$. Stefania Spila Alegiani ${ }^{3} \cdot$ ITA-COVID-19: RAAS inhibitor group}

Stefania Spila Alegiani

stefania.spila@iss.it

1 Department of Biomedical and Dental Sciences and Morphofunctional Imaging, University of Messina, Messina, Italy

2 Italian Society of Pharmacology, Milan, Italy

3 Pharmacoepidemiology Unit, National Centre for Drug Research and Evaluation, Istituto Superiore di Sanità, Viale Regina Elena 299, 00161 Rome, Italy
4 Azienda Unità Sanitaria Locale-IRCCS, Reggio Emilia, Italy

5 Azienda Zero - Department of Health of Veneto Region, Padua, Italy

6 Epidemiology Observatory - Department of Health of Lombardy Region, Milan, Italy 